\title{
EDITORIAL
}

\section{Pediatric Neurotherapy}

To readers familiar with NeuroRx, this issue may represent somewhat of a departure. Typically, each edition focuses on a specific type of disorder, or perhaps considers conditions with a common pathophysiology. However, this month, the focus is on Child Neurology. We have recruited authors with diverse expertise and solicited contributions that share a common theme-therapeutic interventions for neurological disorders in children.

One of the banes of a career as a pediatric neurologist is the frequent comment from other physicians, from medical students, and from casual acquaintances that "it must be so depressing. . . you can't treat anything. ..." Indeed, this perception may be well deserved, given the rarity of many childhood conditions, the scarcity of qualified clinicians to evaluate and diagnose these problems, and the problems inherent in gathering evidence-based clinical data to evaluate therapeutic interventions. However, the contributors to this volume present substantial new information to contradict this perspective. They provide current perspectives about the rapidly broadening scope of therapeutic options for children with many acute and chronic neurological disorders. Moreover, several highlight how new understanding of the fundamental biology of a variety of neurological disorders of childhood will lay the foundation for development of more effective treatment in the foreseeable future.

A wide range of topics was selected for this volume, both to emphasize the challenges inherent in treating childhood neurological disorders and also to illustrate the diverse strategies (developed both in the laboratory and the clinic) that have yielded more effective treatments and a greater understanding of neurobiological mechanisms of disease. We would like to acknowledge Dr. Donna Ferriero's contributions to the initial selection of topics and recruitment of authors.

The first three articles focus on themes related to pediatric neurology emergencies: cerebral ischemia, ${ }^{1}$ traumatic brain injury, ${ }^{2}$ and the role of induced hypothermia to limit progression of acute brain injury in children. ${ }^{3}$ The successful completion of two recent multicenter,

Address correspondence and reprint requests to Leon S. Dure, M.D., Bew White Professor of Pediatrics and Neurology Department of Pediatrics, Division of Child Neurology, The University of Alabama at Birmingham, 1600 7th Avenue South, $314 \mathrm{CH}$ Birmingham, AL 35233 . randomized, controlled trials that demonstrated efficacy of hypothermia in the treatment of neonatal hypoxicischemic encephalopathy underscores the essential role that multicenter collaboration must play in improving treatment of pediatric neurological disorders.

The next five articles focus on common chronic neurological disorders that represent the mainstay of outpatient pediatric neurology practice: epilepsy, ${ }^{4}$ headache, ${ }^{5}$ Tourette syndrome, ${ }^{6}$ autism, ${ }^{7}$ and cerebral palsy. ${ }^{8}$ Important themes include recent discoveries and advances in drug therapy, the necessity for multidisciplinary teams in optimizing treatment, and the potential efficacy of nonmedical interventions in improving overall function. Similarly, each article illuminates the difficulties inherent in the development of clinical trials that address childhood neurologic diseases. The fundamental deficiencies in much of what is known about pediatric headache, tic disorders, epilepsy, etc., are being corrected through efforts described in these articles.

The next group of articles discuss less common pediatric neurological disorders, yet they share the feature of being the subject of extraordinarily informative basic research. Duchenne muscular dystrophy, ${ }^{9}$ spinal muscular atrophy, ${ }^{10}$ adrenoleukodystrophy, ${ }^{11}$ and congenital disorders of glycosylation ${ }^{12}$ are becoming better understood in terms of pathophysiology. Moreover, for each of these disorders the authors highlight how an understanding of underlying molecular mechanisms of disease has provided insights about how to develop more effective treatment.

The final two articles deal with two uncommon (usually sporadic) pediatric neurological disorders, multiple sclerosis (MS) $)^{13}$ and brain tumors. ${ }^{14}$ Pediatric MS has occupied little space in neurologic literature, but this is rapidly changing. Concerted efforts by investigators throughout the world have resulted in consensus regarding terminology and classification of pediatric demyelinating disorders and will be the basis for treatment trials. Similarly, there have been major advances in the treatment of childhood brain tumors-stemming, in large part, from the strategy of enrolling as many affected children as possible in multicenter clinical trials. Progress in this field should serve as an impetus for development of the infrastructure that would facilitate similar multicenter collaborative therapeutic trials for other acute and chronic pediatric neurological disorders. 
In summary, it should be clear to all that child neurologists have often labored in conditions characterized by an incomplete understanding of what they are trying to treat and manage. Much of this is due to the problems inherent in any field concerning children - the diseases are uncommon, the phenotype may be intrinsically related to age of presentation, and the characterization of any disorder is only made more complex in the context of a developing organism. Despite these impediments, progress is occurring, as this issue will prove. We can predict with confidence that such advances will shed more light on the field in general, thus attracting more investigation and research into what we consider a sometimes overlooked frontier.

Leon S. Dure IV, M.D. E-mail: ldure@peds.uab.edu

Faye Silverstein, M.D.

E-mail: fsilvers@med.umich.edu

Guest Editors

\section{REFERENCES}

1. Kirton A, deVeber G. Therapeutic approaches and advances in pediatric stroke. NeuroRx 3:133-142, 2006.
2. Potts MB, Koh S-E, Whetstone WD, Walker BA, Yoneyama T, Claus CP, et al. Traumatic injury to the immature brain: inflammation, oxidative injury, and iron-mediated damage as potential therapeutic targets. NeuroRx 3:143-153, 2006.

3. Gunn AJ, Thoresen M. Hypothermic neuroprotection. NeuroRx 3:154-168, 2006.

4. Donner EJ, Snead OC III. New generation anticonvulsants for the treatment of epilepsy in children. NeuroRx 3:170-180, 2006.

5. Lewis DW. The pharmacological treatment options for pediatric migraine: an evidence-based appraisal. NeuroRx 3:181-191, 2006.

6. Scahill L, Erenberg G, Berlin CM, Jr, Budman C, Coffey BJ, Jankovic J, et al. Contemporary assessment and pharmacotherapy of tourette syndrome. NeuroRx 3:192-206, 2006.

7. Filipek PA, Steinberg-Epstein R, Book TM. Intervention for autistic spectrum disorders. NeuroRx 3:207-216, 2006.

8. Tilton AH. Therapeutic interventions for tone abnormalities in cerebral palsy. NeuroRx 3:217-224, 2006.

9. Strober JB. Therapeutics in Duchenne muscular dystrophy. NeuroRx 3:225-234, 2006.

10. Summer CJ. Therapeutics development for spinal muscular atrophy. NeuroRx 3:235-245, 2006.

11. Moser HW. Therapy of x-linked adrenoleukodystrophy. NeuroRx 3:246-253, 2006.

12. Eklund EA, Freeze HH. The congenital disorders of glycosylation-a multifaceted group of syndromes. NeuroRx 3:254-263, 2006.

13. Chabas D, Green AJ, Waubant E. Pediatric multiple sclerosis. NeuroRx 3:264-275, 2006.

14. Robertson PL. Advances in treatment of pediatric brain tumors. NeuroRx 3:276-291, 2006. 